\title{
Benchmarking Different BAMLET-like Preparations With Respect to Tryptophan Exposure, Interfacial Activity, and Effect on Cell Viability Hanzhen Wen ${ }^{1}$, Ida M. Rundgren ${ }^{1}$, Wilhelm R. Glomm² and Øyvind Halskau ${ }^{1 *}$
}

${ }^{1}$ Department of Molecular Biology, University of Bergen, Thormøhlensgt. 55, 5008 Bergen, Norway

${ }^{2}$ Department of Chemical Engineering, Norwegian University of Science and Technology, N-7491 Trondheim, Norway

\begin{abstract}
Human a-Lactalbumin Made Lethal to Tumors (HAMLET) or its bovine counterpart BAMLET is an $\alpha$-Lactalbumin ( $\alpha$-LA)-oleic acid (OA) complex which induces death to tumor cells, selectively. Although HAMLET or its bovine counterpart BAMLET is prepared chromatographically, it has also been reported that HAMLET or BAMLET-like complexes can be formed by direct mixing of the two components. In this study, we prepare BAMLET-like complexes with reference to the literature, characterize and benchmark these with respect to cytotoxicity, ability to disrupt the integrity of artificial membranes, as well as spectroscopic and physicochemical properties. We show that mixing bovine $\alpha$-Lactalbumin (BLA) with pure OA (liquid form), followed by incubation and shaking at $37^{\circ} \mathrm{C}$ is the most attractive choice for preparing BLA-OA complexes (resulting complex referred to as BLAOA (pure)). Pre-dissolving $O A$ in methanol or ethanol and then mixing with BLA also produces a complex (referred to as BLAOA (methanol) or BLAOA (ethanol)) with similar activities as BAMLET. BLA mixed with OA pre-dispersed in phosphate buffer forms little of the active complex.
\end{abstract}

\begin{abstract}
Abbreviations: $\alpha$-LA, $\alpha$-Lactalbumin; ANTS, 8-Aminonaphtalene1,3,6-trisulfonic acid; BAMLET, Bovine $\alpha$-Lactalbumin Made Lethal to Tumors; BLA, Bovine a-Lactalbumin; EYPC, egg yolk phosphatidylcholine; OA, oleic acid; HAMLET, Human $\alpha$-Lactalbumin Made Lethal to Tumors; HLA, Human a-Lactalbumin; PBS, phosphate buffer saline; PBPS, porcine brain phosphatidylserine; DPX, p-xylenebis-N-pyrimidinum bromide
\end{abstract}

\section{Introduction}

Human $\alpha$-Lactalbumin Made Lethal to Tumors (HAMLET) was first reported as a multimeric oleic acid (OA) containing milk fraction with the ability to selectively kill a wide range of cancer cells in suspension [1]. Since then, this phenomenon has attracted the attention of several laboratories. HAMLET, or its bovine counterpart BAMLET, is prepared by a chromatographic technique, where the calcium-depleted apo-form of $\alpha$-Lactalbumin ( $\alpha$-LA) in the human (HLA) or bovine (BLA) variety is loaded on an anion-exchange column preconditioned with OA. HAMLET/BAMLET can then be eluted at high $(1 \mathrm{M} \mathrm{NaCl})$ salt concentrations [2]. However, others [3-5] have reported that a HAMLET or BAMLET-like complex between $\alpha$-LA and oleic acid can be formed by simple solution mixing of the two components. It was shown [4] that HLA-OA complexes formed by titrating HLA with small aliquots of OA solution in ethanol resemble HAMLET in terms of cytotoxicity. Spolaore et al [3] prepared a BLA-OA complex by simple mixing of BLA and OA in solution (OA was pre-dispersed in phosphate buffer) and the BLA-OA complex displayed cellular toxicity toward Jurkat cells. OA could also be directly suspended into the $\alpha$-LA solution and a tumoricidal complex was produced by heat-treatment [5].

While there are different ways of preparing the complex of $\alpha$-LA and oleic acid, a study characterising and benchmarking these complexes against each other has not been carried out. In this work we prepare BAMLET-like complexes with reference to the literature, focussing on ways of introducing $\mathrm{OA}$ to $\mathrm{BLA}$ at $37^{\circ} \mathrm{C}$ using three different chemical accelerators as well as an emulsification technique. The accelerators were chosen by starting with buffer/water $(\mathrm{H}-\mathrm{OH})$, and increasing the accelerator ability to dissolve OA by going to methanol (Methyl$\mathrm{OH}$ ), and (Ethyl-OH), while still being miscible with the buffer. We characterize and compare these with respect to cytotoxicity, ability to disrupt the integrity of artificial membranes, spectroscopic and physicochemical qualities in order to find an efficient protocol for preparing the complex. We note that there are significant batch-tobatch variability in the preparation of BAMLET-like complexes, offer a straightforward test that can be used as a quick quality control in lieu of or supplementing cell assays, and comment on practical issues for BAMLET-like complexes.

\section{Materials and Methods}

\section{Materials}

Bovine $\alpha$-LActalbumin (BLA, type III, calcium depleted) and oleic acid (OA) were purchased from Sigma. BAMLET was a generous gift from the laboratory of Professor Svanborg, Lund University, Sweden, and prepared as described [2]. Egg yolk phosphatidylcholine (EYPC) and porcine brain phosphatidylserine (PBPS) lipids were from Avanti Polar Lipids, Inc. 8-Aminonaphtalene-1,3,6-trisulfonic acid, disodium salt (ANTS) and p-xylene-bis-N-pyrimidinum bromide (DPX) were from Sigma. All other chemicals were from Merck.

\section{Preparation of the BLA-OA complexes}

BLA was dissolved in phosphate buffer saline (PBS), $\mathrm{pH}$ 7.4, to make stock solutions at $400 \mu \mathrm{M}$. OA was introduced to BLA by the following four methods, three methods employing a chemical accelerator, and the

*Corresponding author: Øyvind Halskau, Department of Molecular Biology, University of Bergen, Thormøhlensgt. 55, 5008 Bergen, Norway, Tel: +47 555845 63; Fax: +47 555896 83; E-mail: oyvind.halskau@mbi.uib.no

Received December 01, 2011; Accepted December 19, 2011; Published December 24, 2011

Citation: Wen H, Rundgren IM, Glomm WR, Halskau $\varnothing$ (2011) Benchmarking Different BAMLET-like Preparations With Respect to Tryptophan Exposure, Interfacial Activity, and Effect on Cell Viability. J Bioanal Biomed S5: 003. doi:10.4172/1948-593X.S5-003

Copyright: (C) 2011 Wen $\mathrm{H}$, et al. This is an open-access article distributed unde the terms of the Creative Commons Attribution License, which permits unrestricted use, distribution, and reproduction in any medium, provided the original author and source are credited. 
Citation: Wen H, Rundgren IM, Glomm WR, Halskau Ø (2011) Benchmarking Different BAMLET-like Preparations With Respect to Tryptophan Exposure, Interfacial Activity, and Effect on Cell Viability. J Bioanal Biomed S5: 003. doi:10.4172/1948-593X.S5-003

fourth adding OA directly to the stock solution of BLA as a pure liquid. For the three methods employing accelerators, a suitable amount of $\mathrm{OA}$ was first dispersed in PBS, methanol or ethanol, as indicated, and this was subsequently mixed with the BLA stock solution to a final BLA/ $\mathrm{OA}$ ratio of $1 / 10$. The resulting three preparations are subsequently referred to as BLAOA(PBS), BLAOA(methanol) and BLAOA(ethanol). The final methanol or ethanol concentration in the mixture is approximately $1 \%$. For BLAOA(PBS), OA was dispersed in phosphate buffer, $\mathrm{pH} 7.4$ and vortexed before mixing with the BLA solution. For the fourth and final preparation, referred to as BLAOA(pure), OA was added to the BLA solution as pure liquid, directly, to reach a BLA/OA ratio of 1/10. Typical amounts of pure OA liquid for $2 \mathrm{~mL}$ BLA stock solution was $2.53 \mu \mathrm{L}$. For BLAOA(PBS) this was predispersed in $2 \mathrm{~mL}$ PBS. For BLAOA(methanol) and BLAOA(ethanol), $50 \mu \mathrm{L}$ of OA stock solution at $154 \mathrm{mM}$ were added to the BLA stock-solution. After OA introduction of any kind, the BLA-concentration in the mixture was adjusted with phosphate buffer to a theoretical concentration of 200 $\mu \mathrm{M}$ in all instances, mixed, and also measured by UV-Vis spectroscopy, see below. All the BLAOA mixtures were subsequently incubated on a shaker for $1 \mathrm{~h}$ at $37^{\circ} \mathrm{C}$. It should be noted that the $\mathrm{OA}$-concentration in the final samples is far above the critical micelle concentration for $\mathrm{OA}$ in aqueous solution $(20 \mu \mathrm{M})[6]$.

\section{Intrinsic fluorescence spectroscopy}

Intrinsic fluorescence measurements at a protein concentration of $4 \mu \mathrm{M}$ in a quartz $10 \mathrm{~mm}$ cuvette were made on a LS50B Perkin Elmer luminescence spectrometer using an excitation wavelength of $295 \mathrm{~nm}$. Emission was scanned at $200 \mathrm{~nm} / \mathrm{min}$ between 310 and $380 \mathrm{~nm}$ for 3 repeats. Both excitation and emission slits were set to $5 \mathrm{~nm}$. 2-3 sample parallels were acquired and blanks were subtracted.

\section{Fluorescence-monitored leakage assays}

Large Unilamellar Vesicles (LUVs) were prepared from EYPC and PBPS [7]. The prerequisite amount of chloroform-dissolved 1:1 mixture of EYPC:PBPS was added to a glass tube wrapped in aluminium foil. The chloroform solution was dried to a thin lipid film using a nitrogen stream and residual chloroform was removed under vacuum for 4 hrs. The sample was then mixed with PBS, pH 7.4, containing $12.5 \mathrm{mM}$ ANTS and $45 \mathrm{mM}$ DPX and left to hydrate overnight on a shaker at $37^{\circ} \mathrm{C}$. To prepare LUVs, the solution was subjected to seven freeze/thaw cycles using liquid nitrogen and a warm water bath. The sample was extruded through a $100 \mathrm{~nm}$ pore-size membrane (Nuclepore,Whatman) 9 times using Avanti Mini-Extruder (Avanti Polar Lipids, Inc.) and transferred to a clean tube. The free ANTS and DPX were removed using a prepacked Sephadex PD-10 column (GE Healthcare Biosciences Corp., NJ).

The leakage assay was conducted, as described by Ellens et al. [8]. ANTS and DPX were used as a fluorophore and quencher, respectively. LUVs containing ANTS/DPX were diluted to $0.6 \mathrm{mM}$ lipid concentration using PBS, pH 7.4. $700 \mu \mathrm{L}$ LUVs were added to a $10 \mathrm{~mm}$ quartz cuvette and fluorescence was measured with LS50B Perkin Elmer luminescence spectrometer (355 nm excitation, 450$550 \mathrm{~nm}$ emission, scan speed of $200 \mathrm{~nm} / \mathrm{min}, 5 \mathrm{~nm}$ slit widths for both excitation and emission). Volumes $(300 \mu \mathrm{L})$ of sample solutions were added to the cuvette ( $700 \mu \mathrm{L}$ LUV solution) with gentle mixing to reach a lipid/protein or lipid/OA molar ratio of $7 / 1$, with protein concentration of $60 \mu \mathrm{M}$. Fluorescence was recorded for each sample at $25{ }^{\circ} \mathrm{C}$. The emission spectra are dominated by ANTS fluorescence $\left(\lambda_{\max }\right.$ at $\left.510 \mathrm{~nm}\right)$. The fluorescence intensity at $\lambda_{\max }$ is closely related to the ANTS/DPX proximity. When the membrane integrity is disturbed, there will be an increase in the fluorescence intensity as ANTS and DPX are released from the LUVs and the average distance between ANTS and DPX increases. Triton X-100 was added to the cuvette at the end to completely break down the LUVs and fluorescence at $510 \mathrm{~nm}$ was arbitrarily set to $100 \%$. Fluorescence at $510 \mathrm{~nm}$ prior to addition of sample stock solution was arbitrarily set to $0 \%$. The values represent the average of 3 parallels.

\section{The ratio of absorbance at $260 \mathrm{~nm}$ and $280 \mathrm{~nm}$}

To assess protein aggregation levels [9], the ratios of absorbance at $260 \mathrm{~nm}$ and $280 \mathrm{~nm}$ for different BLAOA complexes were measured with a NanoDrop1000 Spectrophotometer. The protein concentration of the samples was measured to $170 \mu \mathrm{M}$ [10]. The sample volume was $2 \mu \mathrm{l}$.

\section{Langmuir Isotherms}

The surface pressure-time isotherms of BLA and BLAOA (pure) were measured using a KSV Langmuir trough system (KSV, Finland). The trough is made of Teflon, and the instrument fitted with a Wilhelmy plate for force measurements. Prior to introduction of the sample, the trough was filled with $\mathrm{PBS}, \mathrm{pH} 7.4$, and the surface pressure was set to zero. The pressure was stable for at least 60 seconds. An appropriate amount of sample solution corresponding to a total protein concentration of $3 \mathrm{ppm}(200 \mathrm{nM})$ was injected to the bottom of the trough. The change in surface pressure was then recorded as a function of time at room temperature.

\section{Cell culturing and trypan blue viability assay}

HeLa cells were cultured in Dulbecco's modified eagle's medium with fetal bovine serum ( $10 \%$ of medium volume) and PenicillinStreptomycin $(1 \%)$ at $37{ }^{\circ} \mathrm{C}$ and $5 \% \mathrm{CO}_{2}$ on $10 \mathrm{~mL}$ culture dishes. One $10 \mathrm{~mL}$ dish was used per 12 well plate and three parallels were made for each BLAOA substance tested, and for each control. Prior to plating, the medium was first removed and the cells were washed with $2 \mathrm{~mL}$ of PBS. Approximate $1 \mathrm{~mL}$ of trypsine solution, diluted 1:2 in PBS, was used to detach the cells. Detaching was allowed to proceed for maximum 1 minute before being stopped by addition of fresh medium. A cell suspension was made, and the cells were counted. Typical counts were $7 \cdot 10^{5}$ per $\mathrm{mL}$ and $1 \mathrm{~mL}$ of the cell suspension were transferred to each of the 12 wells. $500 \mu \mathrm{L}$ of BLAOA preparation were added to each well. $5 \mu \mathrm{L}$ of Triton X-100 was added together with $195 \mu \mathrm{L}$ of PBS as a positive control, as well as $200 \mu \mathrm{L}$ of BAMLET. $200 \mu \mathrm{L}$ of BLA and of PBS was added as negative controls. The final protein concentration in each well was $66 \mu \mathrm{M}$.

\section{Results and Discussion}

\section{Intrinsic fluorescence}

HAMLET is known to be in a partially folded, molten globule-like state, with fluctuating tertiary structure [2]. Both human and bovine $a$-La contains four tryptophans that are sensitive to the overall fold of the protein, and can thus be used as probes for measuring changes in the fold induced when preparing HAMLET- and BAMLET-like substance. In this study, we focus on the bovine protein, i.e. BAMLETlike substances. To assess whether different procedures for preparing such substances are prone to random variation, we independently prepared BLAOA(methanol), BLAOA(ethanol), BLAOA(pure), and BLAOA(PBS) 3 times, as described in the Materials and Methods. We also prepared apo-BLA, as well as BAMLET provided by the lab 
Citation: Wen H, Rundgren IM, Glomm WR, Halskau $\varnothing$ (2011) Benchmarking Different BAMLET-like Preparations With Respect to Tryptophan Exposure, Interfacial Activity, and Effect on Cell Viability. J Bioanal Biomed S5: 003. doi:10.4172/1948-593X.S5-003

Page 3 of 5

of Svanborg as references. Thereafter, each sample was characterised by intrinsic tryptophan fluorescence by excitation at $295 \mathrm{~nm}$ and recording of the resulting emission spectrum. Typical spectra are presented in Figure 1a along with spectra of apo-BLA and BAMLET as references. The average $\lambda_{\max }$ and average normalized intensity values based on the independently prepared substances are summarized in Figure $1 \mathrm{~b}$. As can be seen, the most dramatic $\lambda_{\max }$ shifts and intensity increases are seen for BLAOA(pure), which is relatively similar to BAMLET (Figure 1a). The histogram in Figure 1b shows that batchto-batch variation is significant. For instance, the standard deviation of BLAOA(methanol) spans $4 \mathrm{~nm}$, indicating that the exact attributes of a given preparation can vary considerably. However, BLAOA(pure, methanol, and ethanol) show clear red-shifts and intensity increases relative to the unmodified control apo-BLA. In contrast, BLAOA(PBS) has a narrow standard deviation, and is very similar to the control. In this instance, no significant changes appear to have taken place.

\section{Aggregation state and surface activity of complexes}

HAMLET and BAMLET are also invariably described as multimeric, or as amorphous aggregates $[1,11]$. The ratio of light absorbance (A) at $260 \mathrm{~nm}$ and $280 \mathrm{~nm}$ are often used as a rough indicator of the aggregation-state of a protein. UV-Vis spectrometers
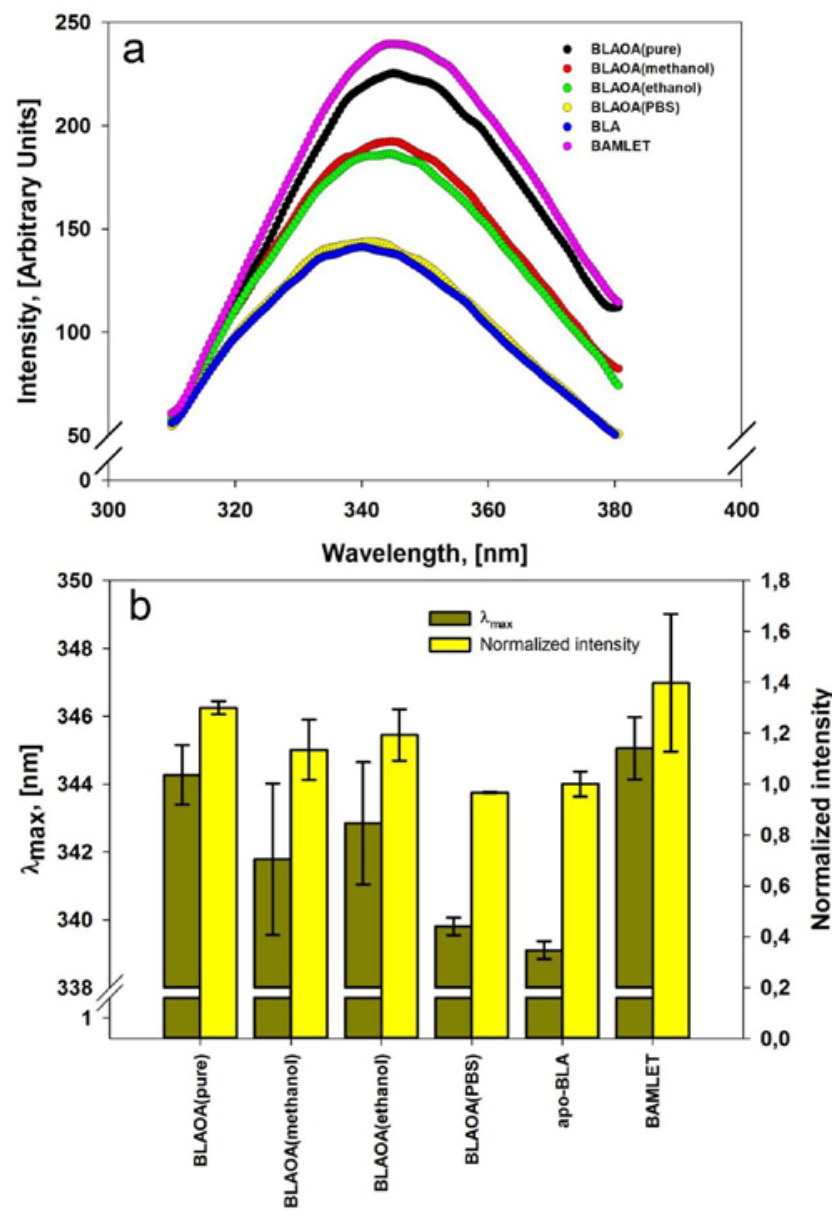

Figure 1: Fluorescence properties of BAMLET and BAMLET-like preparations. a) Representative emission spectra of $\operatorname{BLAOA}($ pure), (•); BLAOA(methanol),

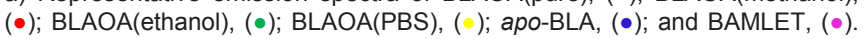
b) Average $\lambda_{\text {max }}$ and normalized intensity-values based on three independently prepared solutions, except for BAMLET, were the results are from three stocksolutions prepared from the same batch of lyophilized powder.
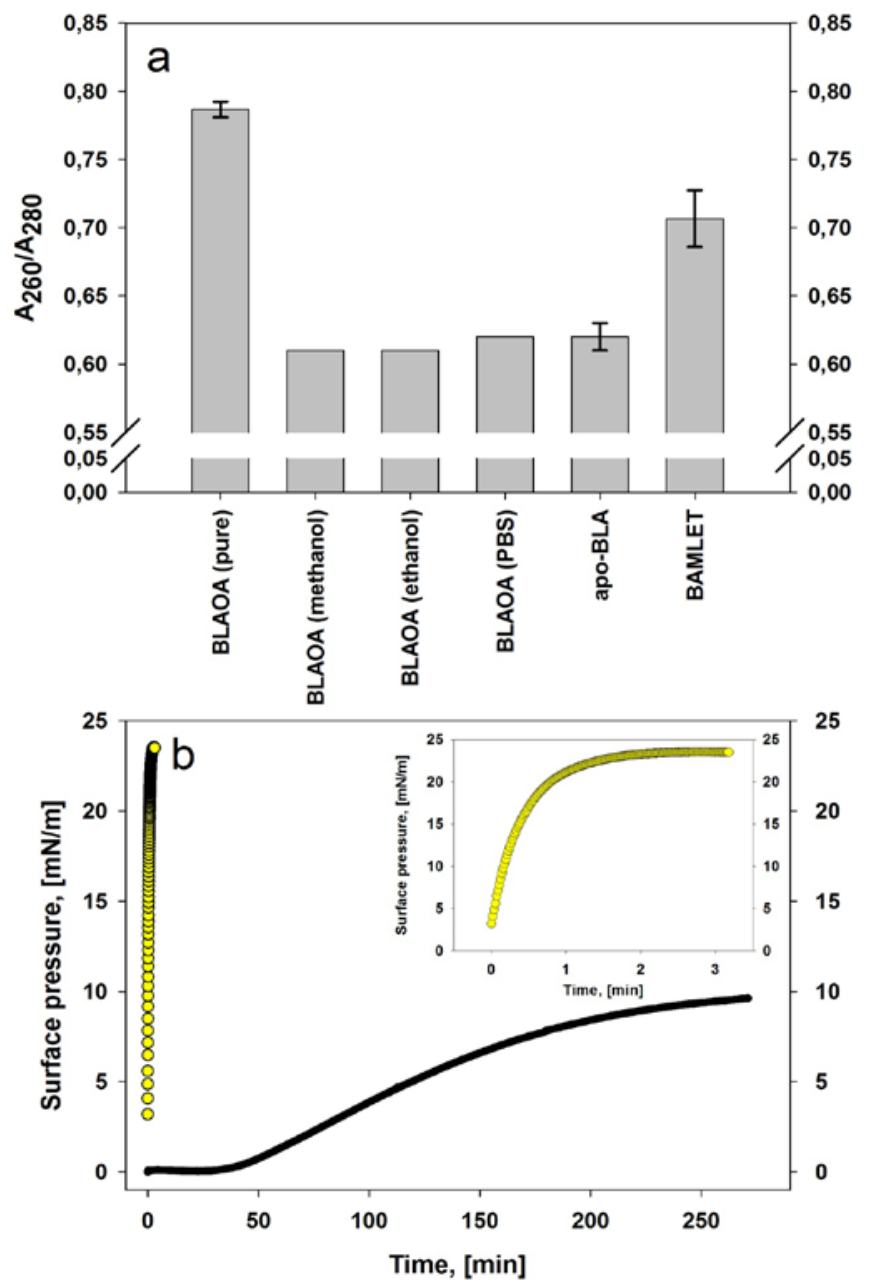

Figure 2: Aggregation-state and surface activity of BAMLET and BAMLET-like preparations. a) Ratio of absorbance at $260 \mathrm{~nm}$ and $280 \mathrm{~nm}$. Average based on three measurements. b) Effect of apo-BLA (•) and $\mathrm{BLAOA}($ pure) ( ) on surface pressure measured by the Langmuir method. Inset: Effect of BLAOA(pure) on surface pressure, timescale zoomed in.

are usually readily available at most labs, and scans are quick to perform. We acquired the spectra and calculated the $\mathrm{A}_{260} / \mathrm{A}_{280} \mathrm{~nm}$ ratios for the range of preparations. As can be seen in Figure 2a, most preparations and apo-BLA have a ratio of around 0.6 which is not high enough to indicate extensive aggregation. In contrast, both BLAOA(pure) and BAMLET has ratios of around 0.8 and 0.7 respectively, suggesting that some aggregation occurs. Overall, there thus appears to be two classes of complexes in our data-set with respect to aggregation, one where aggregation does not occur (BLAOA(PBS), BLAOA(methanol), $\mathrm{BLAOA}($ ethanol)), and one where aggregation is found (BLAOA(pure) and BAMLET).

A characteristic that often follows intermediate folds and aggregation is a heightened surface activity [12]. Since BLAOA(pure) among the preparation studied here was found to display the closest resemblance to BAMLET in terms of emission lineshape and aggregation state, BLAOA(pure) and apo-BLA were investigated for their ability to influence the surface pressure of water using a Langmuir tray fitted with a Wilhelmy plate for exact measurements of forces acting at the interface. Upon injection into the aqueous subphase, both apo-BLA and BLAOA(pure) form Gibbs monolayers at the air-water surface, as 
can be seen in Figure 2b. Introduction of BLAOA(pure) results in a more rapid surface pressure increase as well as a significantly higher $(>2 \mathrm{X})$ final surface pressure than BLA only, revealing the enhanced surface activity of the aggregated BLAOA(pure).

\section{Ability to induce leakage}

Another characteristic of BAMLET related to its surface activity, is its ability to interact with and cause extensive leakage of encapsulated membrane content [13]. This characteristic has been proposed to be part of the earliest steps in the process leading to cell death, and may have bearing on the selectivity of the complex $[14,15]$. Here we use it as a further indicator that BLAOA has formed and has BAMLETlike properties. Briefly, we made vesicle from zwitterionic EYPC and negatively charged PBPS lipid extracts in a 1:1 mol-to-mol mixture, hereafter referred to as EYPC:PBPS. The presence of negative charge appears to be important for efficient leakage, as vesicles made of EYPC only are not susceptible to this effect $[13,14]$. The EYPC:PBPS vesicles were prepared with an encapsulated fluorophore ANTS and quencher DPX. As the complexes interact with and disrupt the bilayer of the vesicle, the contents escape and the leakage can be quantified by measuring the florescence of ANTS. This effect is concentrationdependent [13], but for the purpose of this study we picked one protein concentration of $60 \mu \mathrm{M}$ and compared the ability of the prepared substances to induce leakage. The results are shown in Figure 3. BLAOA(pure) shows the highest ability to cause leakage of ANTS and DPX. BAMLET, BLAOA(ethanol) and BLAOA(methanol) also cause a significant amount of membrane disruption. In contrast, and as for the intrinsic fluorescence results, BLAOA(PBS) is very similar to the controls, apo-BLA and OA, in that it elicits no leakage.

\section{Cell viability assessed by trypan blue exclusion}

Trypan blue is a vital stain excluded by the outer cell membrane. Viable cells remain unstained by this dye, while dead cells with breached membrane will stain blue. HeLa cells are easily cultured, adherent cells that are known to be susceptible to the action of HAMLET and

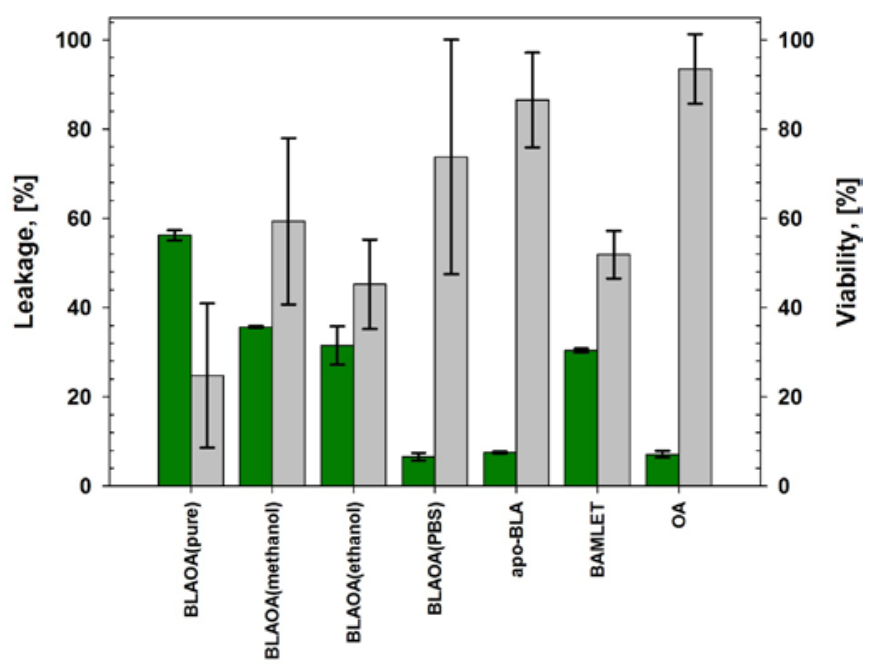

Figure 3: BAMLET and BAMLET-like preparation's ability to cause leakage of EYPC:PBPS vesicles (green bars, left axis) and affect cell viability of HeLa cells (grey bars, right axis). Leakage of vesicular contents were measured by increase in the fluorescence as the fluorophore and quencher pair, ANTS and DPX, escaped and were diluted in the bulk solution upon membrane perturbation. Cell viability was assessed by trypan blue staining and automatic cell counting.
BAMLET [1,2]. HeLa cells were thus cultured, plated out in wells and incubated with the different BLAOA preparations described above, as well as with negative controls (OA, BLA and PBS). The cells were detached and counted using an automated cell counter that can discern stained and unstained cells, as described in the material and methods. The results are presented in Figure 3. BLA and OA themselves have almost no cytotoxicity. Triton X-100 is used as controls. Among all the BLAOA preparations, BLAOA(pure) has the highest cytotoxic effect on the HeLa cells. BLAOA(methanol) and BLAOA(ethanol) have a similar effect as BAMLET on the cells, which is in agreement with a previous report by Knyazeva et al. [4]. BLAOA(PBS) appears to have the least effect.

\section{Methodological differences}

A notable aspect of the results presented here is the consistent lack of response when OA is predispersed in buffer (BLAOA(PBS)) vs. the responses observed for the other accelerators and the BLAOA(pure). The four procedures employed here can be separated into two main categories; solvent-assisted and emulsion-based complex formation. BLAOA(PBS), BLAOA(methanol) and BLAOA(ethanol) can be characterized as solvent-assisted, whereas BLAOA(pure) relies on the thermodynamic compatibility between liquid OA and BLA in the buffer. In the case of the solvent-assisted procedures, the efficacy with which the solvent facilitates transport of OA from the solution phase to BLA confinement may depend critically on the quality of the solvent with respect to $\mathrm{OA}$, and consequently on the aggregation state of $\mathrm{OA}$ upon mixing (i.e.; whether OA exists as monomers or as micelles). In the case of BLAOA(PBS), the solvent quality does not differ from the final bulk state, yet the initial OA concentration is significantly higher, likely resulting in OA self-assembly into micelles or other higher-order structures. Upon self-assembly, the free energy of the system is significantly lowered owing mostly to increased entropy of water molecules, leaving $\mathrm{OA}$ with its headgroups towards the solution, largely eliminating the driving force for BLA-OA complex formation. For BLAOA(methanol) and BLAOA(ethanol), the OA solvency of these accelerators is significantly higher, possibly inhibiting OA selfassembly. If $\mathrm{OA}$ is introduced into the bulk solution as monomers, complexation of OA with BLA will occur, provided the concentration of $\mathrm{OA}$ is above the critical association concentration for the system [16]. Finally, the BLAOA(pure) procedure relies on introducing droplets of pure OA into the buffer, creating a transiently stabilized but thermodynamically unstable emulsion [16]. OA emulsion droplets may eventually disperse from their highly concentrated state and partition into solvent phase as monomers, and associate with BLA via a molecular leaching model from a thermodynamically unstable donor state (OA droplets) to a thermodynamically stable acceptor state (BLA) similar to what is established for emulsion polymerization [16]. We offer these thermodynamical considerations as a possible explanation for the failure of BLAOA (PBS) to form the active complex.

\section{Conclusion}

Considering the data presented above, we find that among BLAOA (pure), BLAOA (methanol), BLAOA (ethanol), and BLAOA (PBS), the viability of BLAOA(PBS)-treated cells is relatively high. Moreover, the fluorescence and leakage data for this preparation is very similar to that of apo-BLA. We conclude that this preparation method forms little of the active complex. The other preparations are more similar to BAMLET, with wells exposed to BLAOA (pure) having the lowest average viability. As can be seen in the standard deviations, the viability assays are subject to relatively large variations, and no absolute 
Citation: Wen H, Rundgren IM, Glomm WR, Halskau $\varnothing$ (2011) Benchmarking Different BAMLET-like Preparations With Respect to Tryptophan Exposure, Interfacial Activity, and Effect on Cell Viability. J Bioanal Biomed S5: 003. doi:10.4172/1948-593X.S5-003

statements about their efficacies should be made based on these alone. Thus, again also considering the fluorescence of the complex, ability to cause leakage and aggregation state, BLAOA (pure) is the preparation most similar to BAMLET. Moreover, as seen by the Langmuir data, the transformation has markedly changed the surface properties of the complex. Preparation of BLAOA (pure) is straightforward and quick, and coupled with the effect on cell viability and reasonable match of other characteristics to BAMLET, it is the most attractive choice for preparing BLAOA complexes.

One problem with working with BLAOA complexes is that independent preparations yield variable results. It is thus recommended to verify formation in each instance by assessing fold and aggregation state. Well-formed BLAOA should have a UV-Vis 260/280 ratio of 0.7 or more, have a marked $\lambda_{\max }$ change relative to apo-BLA $(339 \mathrm{~nm})$ to $343-345 \mathrm{~nm}$, an intensity gain of at least $20 \%$, as well as high surface activity and ability to disrupt membranes. These latter characteristics can be measured in several ways, but a method available to most laboratories is the Langmuir method used here. For active BAMLETlike preparations, the heightened surface activity is associated with the ability to cause leakage (Figure 2, Figure 3, and [13]); the leakage assay is not suitable as a quick test, since the amount of work is similar to that of measuring cell viability.

\section{Acknowledgements}

We would like to thank Prof. Svanborg for providing column-prepared BAMLET.

\section{References}

1. Håkansson A, Zhivotovsky B, Orrenius S, Sabharwal H, Svanborg C (1995) Apoptosis induced by a human milk protein. Proc Natl Acad Sci USA 92: 80648068

2. Svensson M, Håkansson A, Mossberg AK, Linse S, Svanborg C (2000) Conversion of alpha-lactalbumin to a protein inducing apoptosis. Proc Nat Acad Sci USA 97: 4221-4226.

3. Spolaore B, Pinato O, Canton M, Zambonin M, de Laureto PP, et al. (2010) alpha-Lactalbumin forms with oleic acid a high molecular weight complex displaying cytotoxic activity. Biochemistry 49: 8658-8667.
4. Knyazeva EL, Grishchenko VM, Fadeev RS, Akatov VS, Permyakov SE, et al. (2008) Who is Mr.HAMLET? Interaction of human alpha-lactalbumin with monomeric oleic acid. Biochemistry 47: 13127-13137.

5. Kamijimaa T, Ohmuraa A, Satob T, Akimotob K, Itabashi M, et al. (2008) Heat-treatment method for producing fatty acid-bound alpha-lactalbumin that induces tumor cell death. Biochem Biophys Res Commun 376: 211-214.

6. Cistola DP, Hamilton JA, Jackson D, Small DM (1988) Ionization and phase behavior of fatty acids in water: application of the Gibbs phase rule. Biochemistry 27: 1881-1888.

7. Mayer LD, Hope MJ, Cullis PR (1986) Vesicles of variable sizes produced by a rapid extrusion procedure. Biochimica et biophysica acta 858: 161-168.

8. Ellens H, Bentz J, Szoka FC (1984) pH-induced destabilization of phosphatidylethanolamine-containing liposomes: role of bilayer contact. Biochemistry 23: 1532-1538.

9. Ksenofontov AL, Kozlovskii VS, Kordyukova LV, Radyukhin VA, Timofeeva AV (2006) Determination of Concentration and Aggregate Size in Influenza Virus Preparations from True UV Absorption Spectra. Molecular Biology 40: 152-158.

10. Nick PC, Vajdos F, Fee L, Grimsley G, Gray T (1995) How to measure and predict the molar absorption coefficient of a protein. Protein science : a publication of the Protein Society 4: 2411-2423.

11. Zhang M, Yang F, Chen J, Zheng CY, Liang Y (2009) Cytotoxic aggregates of alpha-lactalbumin induced by unsaturated fatty acid induce apoptosis in tumor cells. Chem Biol Interact 180: 131-142.

12. Halskau OA, Muga, Martinez A (2009) Linking new paradigms in protein chemistry to reversible membrane-protein interactions. Curr Protein Pept Sci 10: 339-359.

13. Mossberg AK, Puchades M, Halskau $\varnothing$, Baumann A, Lanekoff I, et al. (2010) HAMLET interacts with lipid membranes and perturbs their structure and integrity. PLoS One 5: e9384.

14. Rødland I, Halskau $\varnothing$, Martínez A, Holmsen H (2005) Alpha-Lactalbumin binding and membrane integrity--effect of charge and degree of unsaturation of glycerophospholipids. Biochim Biophys Acta 1717: 11-20

15. Agasøster AV, Halskau Ø, Fuglebakk E, Frøystein NA, Muga A, et al. (2003) The interaction of peripheral proteins and membranes studied with alphalactalbumin and phospholipid bilayers of various compositions. J Biol Chem 278: $21790-21797$

16. Holmberg K, Jönsson B, Kronberg B, Lindman B (2003) Surfactants and polymers in aqueous solution. 2nd ed2004, West Sussex: John Wiley \& Sons Ltd. 